\title{
Saúde do profissional de Enfermagem: riscos ocupacionais em ambiente hospitalar
}

\section{Nursing professional's health: occupational risks in a hospital environment}

\author{
Carlos Victor Peixoto Dias, Jaiana Cordeiro Damasceno, \\ Lucas Vinícius Farias Silva, Bruno Miranda da Rocha
}

Como citar este artigo: DIAS, CARLOS V. P.; DAMASCENO, JAIANA C.; SILVA, LUCAS V. F.; ROCHA, BRUNO M.

Saúde do profissional de Enfermagem: riscos ocupacionais em ambiente hospitalar. Revista Saúde (Sta. Maria). 2020; 46 (2).

Autor correspondente:

Nome: Carlos Victor Peixoto Dias E-mail: carlosvictorsk8@gmail.com Telefone: (95) 98104-2633

Formação Profissional: Graduando no curso de Bacharelado em Enfermagem pela Universidade Estadual de Roraima (UERR), Boa Vista, RR, Brasil.

Filiação Institucional: Universidade Estadual de Roraima

Endereço para correspondência:

Rua das Margaridas

Bairro: Pricumã

Cidade: Boa Vista

Estado: Roraima

CEP: 69309-550

Data de Submissão:

28/09/20 18

Data de aceite:

28/07/2020

Conflito de Interesse: Não há conflito de interesse

\section{(cc) $\mathrm{BY}-\mathrm{NC}-\mathrm{ND}$}

\section{RESUMO}

Trata-se de uma Revisão Integrativa que teve como descritores não controlados: saúde ocupacional; enfermagem; ambiente hospitalar. Entre os profissionais da saúde, um dos que está mais exposto a riscos é o profissional de enfermagem, uma vez que ele é a pessoa que mais interage com os pacientes para cuidados com a saúde e está exposto a materiais biológicos contaminantes. 0 artigo tem 0 propósito de conhecer e analisar a produção científica em relação a saúde ocupacional e os fatores de risco vivenciados pelos profissionais de enfermagem no ambiente hospitalar. Como estratégia de busca foi elaborada a pesquisa nas bases de dados Scientific Electronic Library Online, Literatura Latino-Americana e do Caribe em Ciências da Saúde e Medical Literature Analysis and Retrieval System Online. Este estudo possibilitou conhecer a produção científica em relação a quais riscos ocupacionais os profissionais de enfermagem estão mais sujeitos no ambiente hospitalar, porém, acredita-se na necessidade de mais estudos e pesquisas referentes a este tema, visto que a literatura ainda é muito carente deste conteúdo

PALAVRAS-CHAVE: Saúde Ocupacional; Enfermagem; Ambiente Hospitalar.

\section{ABSTRACT}

It is an Integrative Review that had as uncontrolled descriptors: occupational health; nursing; hospital environment. Among health professionals, one of those who is most exposed to risks is the nursing professional, since he is the person who most interacts with patients for health care and is exposed to contaminating biological materials. The article aims to know and analyze the scientific production in relation to occupational health and the risk factors experienced by nursing professionals in the hospital environment. As a search strategy, research was conducted in the Scientific Electronic Library Online, Latin American and Caribbean Literature in Health Sciences and Medical Literature Analysis and Retrieval System Online databases. This study made it possible to know the scientific production in relation to which occupational risks nursing professionals are most subject to in the hospital environment, however, it is believed that more studies and research on this topic are needed, since the literature is still very lacking in this area content.

KEYWORDS: Occupational Health; Nursing; Hospital Environment. 


\section{INTRODUÇÃO}

A Saúde Ocupacional possui grande importância no contexto mundial e nacional, em virtude, principalmente, do impacto que as doenças possuem na vida dos trabalhadores e do grande número de acidentes que acometem os empregados. Os acidentes de trabalho, causados pela ruptura da relação entre saúde e trabalho e que interferem no processo saúde/doença do funcionário, são prejudiciais à saúde dos laborais em decorrência da interferência negativa no aspecto físico e psíquico do trabalhador e no contexto social, econômico e político ${ }^{1}$. Segundo o Anuário Estatístico de Acidentes de Trabalho de 2015 (AEAT 2015), foram registrados, nesse ano, 612.632 acidentes de trabalho, sendo 2.502 mortes $^{2}$. Portanto, existir uma saúde ocupacional de alta eficácia é de extrema importância para que os números de casos de acidente de trabalho diminuam cada vez mais.

A Saúde Ocupacional possui alguns anos de história. Essa área da saúde surgiu na Inglaterra, no século XVIII durante a Revolução Industrial, quando existiam péssimas condições de trabalho e os funcionários sentiram a necessidade de reivindicar por uma organização que fiscalizasse os riscos de acidentes ou de adoecimentos que existiam para que pudessem ser evitados ${ }^{3}$. A partir daí médicos começaram a ser contratados para vigiar e proteger a saúde dos funcionários no trabalho. Todavia, esses cuidados visavam apenas a continuidade da produtividade dos trabalhadores e não a integralidade da saúde das pessoas, o que acabou culminando em uma Medicina do Trabalho baseada nos princípios biologicistas e assistencialistas da época. Na primeira metade do século XX, novos problemas e reinvindicações surgiram, o que acabou criando mais espaço para novas profissões participarem da equipe de Medicina do Trabalho para que ocorresse uma abordagem multiprofissional, consolidando-se, assim, a Saúde Ocupacional ${ }^{4}$.

Após esse período, influenciado por outros países do mundo, o Brasil adotou as práticas assistencialistas e criou os Serviços Especializados em Engenharia de Segurança e Medicina do Trabalho nos anos de 1970. Na década de 1980, iniciou-se o Movimento da Reforma Sanitária que trouxe reivindicações dos trabalhadores em relação ao direito à saúde e ao atendimento com atenção integral à saúde e por um trabalho digno e saudável. Com isso, o termo Saúde do Trabalhador começou a ser amplificado e valorizado nas políticas públicas que surgiram na década de 1980, visto que ela articulava diferentes abordagens para a compreensão do processo trabalho-saúde-doença, como a epidemiologia, a saúde coletiva, a psicologia social, a psicodinâmica do trabalho e as concepções da ergonomia franco-belga, entre outras ${ }^{4}$.

Dessa forma, percebe-se que existem diferenças marcantes entre a Medicina Ocupacional e a Saúde do Trabalhador, sendo que uma surgiu depois que a outra e trouxe uma abordagem mais ampla de cuidado com a saúde dos trabalhadores. A Saúde Ocupacional tinha foco em manter o trabalhador seguro e evitar doenças ou acidentes, limitandose apenas ao ambiente de trabalho ${ }^{5}$. De outro lado, a Saúde do Trabalhador considera a doença como algo multifocal e 
leva em consideração também a opinião dos trabalhadores para que seja possível oferecer uma qualidade de vida maior a essas pessoas, prezando pela qualidade de vida delas.

Todavia, a Saúde Ocupacional é muito importante em diversos ambientes, principalmente no hospitais. Esses lugares podem oferecer diversos riscos para os profissionais de saúde, em virtude de haver altas chances de contaminação por doenças, de acidentes com materiais cortantes ou químicos, além dos aspectos psicológicos dos funcionários, afetados principalmente pelas longas e estressantes jornadas de trabalho(6). Além disso, de acordo com um levantamento feito nos anos de 2012 até 2016, pelo Ministério Público do Trabalho, as atividades realizadas no atendimento hospitalar possuem os maiores índices de acidente no trabalho, quando comparadas com outras áreas de trabalho7.

Entre os profissionais da saúde, um dos que está mais exposto a riscos é o profissional de enfermagem, uma vez que ele é a pessoa que mais interage com os pacientes para cuidados com a saúde e está exposto a materiais biológicos contaminantes, além de lidar constantemente com instrumentos perfurocortantes ${ }^{(5)}$. Os enfermeiros também são afetados pelo estresse ocupacional e, segundo uma pesquisa sobre estresse ocupacional na enfermagem, cerca de $36 \%$ dos profissionais de enfermagem estão insatisfeitos com a qualidade de vida no trabalho, principalmente em virtude do baixo apoio social no ambiente laboral, ao baixo controle sobre o trabalho e a alta demanda psicológica por lidar constantemente com pessoas em condições de doença. Sendo assim, percebe-se a importância do estudo em relação aos riscos ocupacionais em profissionais de enfermagem no ambiente hospitalar ${ }^{8}$.

Diante ao referido tem-se como propósito de pesquisa conhecer e analisar a produção científica em relação a saúde ocupacional e os fatores de risco vivenciados pelos profissionais de enfermagem no ambiente hospitalar.

\section{MÉTODO}

Esta pesquisa possui características descritivas com abordagem quantitativa, realizada por meio do método de Revisão Integrativa. Esse método tem a finalidade de reunir e sintetizar resultados de pesquisas sobre um delimitado tema ou questão, de maneira sistemática e ordenada, contribuindo para o aprofundamento do conhecimento do tema investigado ${ }^{6}$. A análise dos dados foi realizada com base nos estudos de Whittemore e Knafl9; e Russell ${ }^{10}$.

O processo de revisão integrativa em cinco etapas inclui (1) formulação de problemas, (2) coleta de dados ou pesquisa na literatura, (3) avaliação de dados, (4) análise de dados e (5) interpretação e apresentação dos resultados.

Foi utilizada a seguinte questão norteadora: quais riscos ocupacionais os profissionais de enfermagem estão mais sujeitos no ambiente hospitalar? 
Buscando na literatura científica, realizou-se via online a busca de artigos relacionados ao tema proposto. Como estratégia de busca foi elaborado a pesquisa nas bases de dados eletrônicas do Centro Latino-Americano e do Caribe de Informações em Ciências da Saúde (LILACS), Base de Dados Especializada em Ciências Biomédicas e Ciências da Vida (MEDLINE) e Scientific Electronic Library Online (SCIELO).

Foram utilizados os descritores não controlados Saúde Ocupacional; Enfermagem, e Ambiente Hospitalar. Após realizar a busca com o termo Saúde Ocupacional acrescentamos o operador booleano and para incluir a palavra Enfermagem; na terceira busca utilizamos o operador booleano and para acrescentar o termo ambiente hospitalar.

Para a seleção dos artigos recorreu-se aos seguintes critérios de inclusão: ser artigo escrito em português, texto completo, disponível online gratuitamente e que corresponda a temática em estudo. Estabeleceu-se como critérios de exclusão: não utilizar textos incompletos e artigos que não estivessem disponíveis na íntegra on-line.

A busca e a seleção dos artigos incluídos na revisão foram realizadas por dois revisores de forma independente.

Inicialmente, ao utilizar o descritor saúde ocupacional, obteve-se 8.160 artigos na base de dados LILACS, 106.854 artigos na MEDLINE e 1.071 artigos na SCIELO, totalizando 116.085 artigos. Filtrando ainda mais os resultados utilizando acrescentando como descritor enfermagem, foram encontrados 847 artigos no LILACS, 4.261 artigos na MEDLINE e 122 artigos na SCIELO, somando um total de 5.230 artigos. Adicionando o descritor ambiente hospitalar, obteve-se na LILACS 58 artigos, na MEDLINE 29 artigos e na SCIELO 6 artigos, gerando um total de 93 artigos.

A análise inicial desses artigos indicou que 6 artigos preenchiam os critérios estabelecidos, sendo os mesmos incluídos neste estudo. Para melhor visualização dos dados obtidos por essa busca investigativa, foi construído um quadro contendo os seguintes itens: $n^{0}$ do artigo, ano, periódico, título, abordagem metodológica, base de dados e objetivos.

A pesquisa levou em consideração os aspectos éticos, respeitando a autoria das ideias, os conceitos e as definições presentes nos artigos incluídos na revisão.

\section{RESULTADOS}

Dos estudos selecionados, a pesquisa pela temática abordou um total de 6 artigos selecionados conforme a Tabela 1, organizados pelo número do artigo, ano/base de dados em ordem crescente, periódico, título, método e objetivos de cada instrumento. Os resultados foram organizados por categorias sendo compreendidos por 1 artigo utilizando a base de dados SCIELO, 2 artigos como base de dados LILACS e 3 artigos como base de dados MEDLINE. 
Tabela 1: Estudos digitais que apresentaram instrumentos para avaliar A Saúde do profissional de enfermagem relacionando aos riscos ocupacionais em ambiente hospitalar.

\begin{tabular}{|c|c|c|c|c|c|}
\hline Artigo & $\begin{array}{l}\text { Ano/Base } \\
\text { de dados }\end{array}$ & Periódico & Título & Método & Objetivos \\
\hline 1 & $\begin{array}{c}2009 \\
\text { MEDLINE }\end{array}$ & $\begin{array}{l}\text { Rev. de } \\
\text { pesquisa: } \\
\text { Cuidado é } \\
\text { Fundamental } \\
\text { Online }\end{array}$ & $\begin{array}{l}\text { A produção do } \\
\text { conhecimento dos } \\
\text { enfermeiros em saúde } \\
\text { do trabalhador acerca } \\
\text { do cenário hospitalar }\end{array}$ & Descritiva & $\begin{array}{l}\text { Relacionar em literatura científica a produção } \\
\text { do conhecimento de enfermagem em saúde } \\
\text { do trabalhador no âmbito hospitalar; e } \\
\text { discutir os principais temas abordados pela } \\
\text { enfermagem em saúde do trabalhador, no } \\
\text { âmbito hospitalar. }\end{array}$ \\
\hline 2 & $\begin{array}{l}2010 \\
\text { LILACS }\end{array}$ & $\begin{array}{l}\text { Esc Anna Nery } \\
\text { Rev Enferm }\end{array}$ & $\begin{array}{l}\text { Repercussões } \\
\text { psicofísicas na saúde } \\
\text { dos enfermeiros } \\
\text { da adaptação e } \\
\text { improvisação de } \\
\text { materiais hospitalares }\end{array}$ & $\begin{array}{l}\text { Qualitativa } \\
\text {-Descritiva }\end{array}$ & $\begin{array}{l}\text { Analisar os sentimentos dos enfermeiros } \\
\text { diante da necessidade de adaptação e de } \\
\text { improvisação de materiais e de equipamentos } \\
\text { no ambiente hospitalar e discutir as } \\
\text { repercussões na saúde do enfermeiro diante } \\
\text { desta necessidade. }\end{array}$ \\
\hline 3 & $\begin{array}{c}2012 \\
\text { MEDLINE }\end{array}$ & Esc. Anna Nery & $\begin{array}{l}\text { Prazer e sofrimento } \\
\text { no exercício gerencial } \\
\text { do enfermeiro no } \\
\text { contexto hospitalar }\end{array}$ & Qualitativa & $\begin{array}{l}\text { Analisar os fatores de prazer e sofrimento } \\
\text { no exercício gerencial do enfermeiro no } \\
\text { contexto hospitalar. }\end{array}$ \\
\hline 4 & $\begin{array}{c}2013 \\
\text { LILACS }\end{array}$ & $\begin{array}{l}\text { O Mundo } \\
\text { da Saúde }\end{array}$ & $\begin{array}{l}\text { Fatores de risco } \\
\text { para acidentes com } \\
\text { materiais perfuro } \\
\text { cortantes }\end{array}$ & Exploratório & $\begin{array}{l}\text { Identificar os fatores de risco para acidentes } \\
\text { com material perfuro cortantes e analisar as } \\
\text { principais causas referidas. }\end{array}$ \\
\hline 5 & $\begin{array}{c}2014 \\
\text { SCIELO }\end{array}$ & $\begin{array}{c}\text { Rev. brasileira } \\
\text { de }\end{array}$ & $\begin{array}{l}\text { Agravos à saúde } \\
\text { referidos pelos } \\
\text { trabalhadores de } \\
\text { enfermagem em um } \\
\text { hospital público da } \\
\text { Bahia } \\
\end{array}$ & $\begin{array}{c}\text { Exploratória - } \\
\text { Descritiva }\end{array}$ & $\begin{array}{l}\text { Estimar a ocorrência de agravos à saúde } \\
\text { referidos pelos trabalhadores de enfermagem } \\
\text { em um hospital na Bahia. }\end{array}$ \\
\hline 6 & $\begin{array}{c}2016 \\
\text { MEDLINE }\end{array}$ & Rev. de & $\begin{array}{c}\text { Prevalência } \\
\text { dos sintomas } \\
\text { osteomusculares } \\
\text { relacionados } \\
\text { ao trabalho de } \\
\text { enfermagem no } \\
\text { âmbito hospitalar }\end{array}$ & Quantitativa & $\begin{array}{l}\text { Verificar a prevalência de sintomas } \\
\text { osteomusculares entre os trabalhadores de } \\
\text { enfermagem de um ospital público do estado } \\
\text { do Piauí. }\end{array}$ \\
\hline
\end{tabular}

Fonte: Elaborada pelo autor 


\section{DISCUSSÃO}

Percebe-se que é de suma importância a saúde ocupacional para dos trabalhadores com ênfase para os profissionais da saúde aos quais diretamente estão prestando cuidado e envolvidos em atividades gerenciais. 0 enfermeiro é o gerente do cuidado por essência. Conciliar a gerência do cuidado com a gerência organizacional é fator que pode sobrecarregar esse profissional ${ }^{11}$. Dentre os artigos achados, observou-se a incidência de acidentes acometendo a equipe de enfermagem, em especial os enfermeiros ${ }^{5,12}$.

Os profissionais da área da saúde mais precisamente os enfermeiros estão expostos a muitas doenças devido a sua jornada de trabalho. Deve-se ter uma atenção em especial para sua saúde ocupacional afim de evitar que estes casos se tornem recorrentes no âmbito profissional.

Em um estudo realizado numa instituição hospitalar de Lisboa verificou-se que os enfermeiros são a classe profissional que regista maior número de acidentes, sendo os profissionais que desempenham funções há mais de 10 anos o grupo com maior incidência de acidentes. Dentro dos traumatismos, as lombalgias são o tipo de lesão mais recorrente, principalmente entre assistentes operacionais e enfermeiros ${ }^{6}$.

\section{Fatores que interferem na saúde ocupacional do enfermeiro}

Na atividade profissional ativa dos enfermeiros percebemos uma sobrecarga física, psíquica e social. Asobrecarga de trabalho, expressa pela abrangência das atividades, pela responsabilidade em tomar decisões e pela necessidade de conciliar o cuidado direto ao paciente com atividades gerenciais, foi um fator de sofrimento citados pelos enfermeiros ${ }^{11}$.

Em relação a contrair doenças no ambiente de trabalho, os profissionais mais expostos ao risco de contrair HIV, por exemplo, são aqueles envolvidos na atenção direta aos pacientes. Esse grupo é composto principalmente pelas equipes de enfermagem e médica, em virtude do contato direto e frequente com sangue e secreções ${ }^{5}$.

Existe uma unanimidade entre os enfermeiros ao opinarem sobre a relevância da adaptação e da improvisação de materiais e equipamentos quando o contexto é de precarização das condições de trabalho, o qual conduz a uma insuficiência e/ou inadequação dos insumos hospitalares ${ }^{13}$.

Soma-se a isso o frequente déficit de profissionais nas unidades, os turnos prolongados, as condições inadequadas de trabalho, limitado poder de decisão, entre outros fatores que contribuem para o processo de desgaste destes trabalhadores. Na atual realidade econômica dos trabalhadores da saúde é comum a dupla e até tripla jornada de trabalho, em virtude dos baixos salários, os quais são insuficientes para o sustento adequado da família ${ }^{12}$. 0 efeito da dupla jornada na saúde destes profissionais é marcante, em especial entre as mulheres, que tendem a agregar a realização do trabalho doméstico e o cuidado com os filhos. 
Quanto aos acidentes com pérfurocortantes, que estão entre os mais comuns, a negligência dos profissionais como seja, deixar a agulha desprotegida em lugares indevidos após a sua utilização e o reencapsulamento de agulhas usadas são comportamentos que violam as regras de prevenção. Em determinadas situações, como seja, a agressividade e agitação por parte dos doentes torna necessária6.

Aadesão às precauções nas atividades laborais é a principal estratégia de proteção ao trabalhador na exposição a patógenos transmissíveis, assim como na proteção ao paciente. Porém, a adesão encontra-se abaixo do recomendado 5 .

\section{Principais consequências do ambiente ocupacional sob a saúde do enfermeiro}

A identificação de inúmeros agravos à saúde, referidos pela equipe de enfermagem, são decorrentes das cargas presentes no ambiente de trabalho. A enfermagem é conhecida por desenvolver uma atividade desgastante, que lida diretamente com o sofrimento, exigindo assim, grande desempenho físico e emocional do trabalhador.

As condições de trabalho acabam por influenciar o profissional no que tange o funcionamento psíquico, físico e social. Os sentimentos de sofrimento e de desgaste emocional foram relacionados às condições materiais no trabalho, ao gasto de tempo, ao esforço físico e mental que os enfermeiros vivenciavam para conseguirem adaptar e improvisar os materiais. Também se relacionavam ao medo acerca da qualidade do cuidado que estavam realizando ${ }^{13}$.

Com uma elevada carga horária de trabalho temos o aparecimento de desgastes sendo fator primordial para 0 aparecimento de doenças como por exemplo as osteomusculares. O elevado percentual de referência a sintomas musculoesqueléticos configura-se como relevante problema de saúde entre os profissionais de enfermagem, afetando sua qualidade de vida bem como o desenvolvimento de suas atividades. Sabe-se que a profissão de enfermagem exige muito do sistema osteomuscular dos trabalhadores e frequentemente os profissionais se submetem ao exercício de posturas incorretas o que compromete a coluna vertebral. Características da estrutura física da instituição, como leitos e bancadas baixos, armários altos, entre outros, podem fazer com que a região lombar da coluna vertebral seja a mais afetada ${ }^{14}$.

Trabalhar com o cuidado exige também cuidado para com esse profissional de saúde, para que ele possa desenvolver suas funções com segurança, mesmo que as condições de trabalho nem sempre estejam favoráveis ${ }^{15}$.

Uma vez que o bem-estar e qualidade de vida no trabalho são fatores que influenciam a qualidade final da assistência de enfermagem, os profissionais devem se preocupar em valorizar o ser e o fazer da enfermagem, proporcionando uma qualidade de vida no trabalho ${ }^{16}$.

A insatisfação de trabalhadores da enfermagem com a qualidade de vida no trabalho está associada a três fatores importantes: tabagismo; a atuação em unidades de assistência a pacientes altamente dependentes ou críticos; 0 baixo apoio social no trabalho e os trabalhos considerados ativos e de alta exigênciå. 
Considerando a complexidade desta temática e de sua importância nos ambientes de saúde, sugere-se a necessidade de mais estudos e pesquisas referentes a este tema afim de obter novas alternativas que possam melhorar a qualidade de vida dos enfermeiros implementando medidas para diminuir cada vez mais os riscos ocupacionais nesta classe profissional, visto que, na literatura este conteúdo é muito escasso.

\section{Elaboração de propostas para melhoria do ambiente ocupacional de enfermagem}

Em relação a saúde dos profissionais de enfermagem em especial aos enfermeiros, é perceptível entre eles o não interesse em elaborar uma proposta de saúde do trabalhador voltado para a própria classe, dando foco somente para a análise dos problemas (identificação dos problemas), suas consequências e principalmente suas complicações.

Na medida em que forem sendo implementadas essas propostas, consequentemente a qualidade de vida do profissional tenderia a aumentar, podendo melhorar assim, a comunicação entre as equipes, diminuindo acidentes por instrumentos perfurocortantes, elaborando estratégias para o controle de ansiedade e estresse, riscos ergonômicos no processo de trabalho, riscos posturais, ficando, portanto, evidente a necessidade de ações voltadas para a prevenção e controle desses distúrbios e por fim estratégias para os riscos psíquicos devido a ampla jornada de trabalho.

De acordo com a pesquisa, é notório que existe uma escassez em relação aos estudos voltados a saúde ocupacional dos enfermeiros, tendo achado um total de seis (6) artigos abordando o tema. As possibilidades de pesquisa são muitas dado a falta de conhecimentos que envolvem a temática e principalmente em relação as informações referentes ao estabelecimento de políticas formais propiciadoras de um ambiente de trabalho mais seguro para os profissionais.

Em relação as limitações do estudo, a primeira foi a metodologia utilizada. Utilizamos o método de revisão integrativa. Por ser utilizado descritores muito específicos, a pesquisa se limitou a poucos artigos e consequentemente a poucas informações sobre o tema. A segunda limitação diz respeito ao uso de filtros durante a pesquisa nas bases de dados reduzindo ainda mais a quantidade de artigos relacionados ao nosso tema. A última limitação refere-se a escassez de trabalhos tanto na região norte quanto em outras regiões do país relatando sobre a Norma Regulamentadora 07 Programa de Controle Médico de Saúde Ocupacional.

\section{CONSIDERAÇÕES FINAIS}

Consideramos que o trabalho é um elemento fundamental para a saúde das pessoas e, desde que seja realizado em condições saudáveis, promove sensação de bem-estar, refletindo na melhoria das condições de trabalho e na assistência de enfermagem prestada e consequentemente na qualidade de vida de seus trabalhadores. Uma vez que bem-estar e qualidade de vida no trabalho são fatores que influenciam a qualidade final da assistência de enfermagem. 
Como profissionais, temos que nos preocupar em valorizar o ser e o fazer da enfermagem, proporcionando uma qualidade de vida no trabalho. A realidade das condições de trabalho relacionadas principalmente à precariedade nos hospitais, sejam públicos ou privados, faz com que o enfermeiro apresente mais sofrimento que prazer durante a sua jornada de trabalho, somatizando com uma falta de incentivo para se exercer sua profissão dignamente. Diante disto tem o aparecimento de doenças psicológicas e patologias em geral.

Portanto, podemos constatar que os riscos ocupacionais mais frequentes entre os profissionais de enfermagem no período 2009 e 2018 segundo as literaturas selecionadas temos as doenças osteomusculares causadas devido a grandes cargas de trabalho e esforços físicos elevados, com estes esforços também se nota desequilíbrios psicológicos onde há o aparecimento de síndromes como por exemplo a síndrome de bournout a qual está em evidencia entre os trabalhadores de enfermagem. Com isso podemos notar que há uma falta de atenção para a saúde do profissional de enfermagem e diante disto uma falta de dados a serem analisados para a construção de materiais científicos.

\section{REFERÊNCIAS}

1. Cavalcante CAA, Cossi MS, Costa RRO, Medeiros SM e Menezes RMP. Análise crítica dos acidentes de trabalho no Brasil. Rev. de Atenção à Saúde, v. 13, no 44, abr.jun. 2015, p. 100-109. DOI: 10.13037/rbcs.vol13n44.2681.

2. Brasil. Anuário Estatístico de Acidentes de Trabalho 2015. Disponível em: <http://sa.previdencia.gov.br/ site/2017/05/aeat15.pdf>. Acesso em: 13 de setembro de 2018.

3. Dias EC, Hoefel MG. O desafio de implementar as ações de saúde do trabalhador no SUS: a estratégia da RENAST. Ciência \& Saúde Coletiva, 10(4):817-828, 2005. Disponível em: <http://www.scielo.br/scielo.php?pid=S1413-81232005000400007\&script=sci_abstract\&tlng=pt>. Acesso em: 14 de setembro de 2018. DOI: http://dx.doi. org/10.1590/S1413-81232005000400007.

4. Daldon MTB, Lancman S. Vigilância em Saúde do Trabalhador: rumos e incertezas. Rev. bras. Saúde ocup., São Paulo, 38 (127): 92-106, 2013. DOI: http://dx.doi.org/10.1590/S0303-76572013000100012. Disponível em: <http://www.scielo.br/pdf/rbso/v38n127/v38n127a12.pdf>. Acesso em 14 de setembro de 2018. 
5. Lima KM, Canela KGS, Teles RBA, Melo DEB, Belfort LRM, Martins VHS. Gestão na saúde ocupacional: importância da investigação de acidentes e incidentes de trabalho em serviços de saúde. Rev Bras Med Trab.2017;15(3):276-283. Disponível em: <http://www.rbmt.org.br/details/259/pt-BR/gestao-na-saude-ocupacional--importancia-da-investigacao-de-acidentes-e-incidentes-de-trabalho-em-servicos-de-saude>. Acesso em 14 de setembro de 2018. DOI: 10.5327/Z1679443520173016

6. Mendes T, Areosa J. Acidentes de trabalho ocorridos em profissionais de saúde numa instituição hospitalar de Lisboa, Revista Angolana de Sociologia, 13| 2014, 25-47. Disponível em: <https: //journals.openedition.org/ ras/970>. Acesso em 14 de setembro de 2018. DOI: 10.4000/ras.970

7. Brasil. Ministério Público do Trabalho. Observatório Digital de Saúde e Segurança do Trabalho, 1: 1-3. 2017. Disponível em: <encurtador.com.br/etM37>. Acesso em 14 de setembro de 2018.

8. Azevedo BDS, Nery AA, Cardoso JP. Estresse ocupacional e insatisfação com a qualidade de vida no trabalho da enfermagem. Texto Contexto Enferm, 2017; 26(1):e3940015. Disponível em: <http://www.scielo.br/pdf/tce/ v26n1/pt_1980-265X-tce-26-01-e3940015.pdf>. Acesso em: 14 de setembro de 2018.

9. Whittemore R, Knafl K. The integrative review: updated methodology. J Adv Nurs 2005;52(5):546-53. DOI: 10.1111 / j.1365-2648.2005.03621.x

10. Russell CL. Uma visão geral da revisão integrativa de pesquisa. Prog Transplant. Mar 2005; 15 (1): 8-13.

11. Santos JLG, Prochnow AG, Silva DC, Silva RM, Leite JL e Erdmann AL. Prazer e sofrimento no exercício gerencial do enfermeiro no contexto hospitalar. Esc. Anna Nery 2013, vol.17, n.1, pp.97-103. Disponível em: <http:// www.scielo.br/scielo.php?pid=S1414-81452013000100014\&script=sci_abstract\&tlng=pt>. Acesso em: 28 de setembro de 2018. DOI: http://dx.doi.org/10.1590/S1414-81452013000100014.

12. Machado LFS, Rodrigues EP, Oliveira LMM, Laudano RCS e Sobrinho CLN. Agravos à saúde referidos pelos trabalhadores de enfermagem em um hospital público da Bahia. Rev. bras. enferm. vol.67 no.5 Brasília Sept./Oct. 2014. Disponível em: <http://www.scielo.br/scielo.php?pid=S0034-71672014000500684\&script=sci_abstract>. Acesso em: 28 de setembro 2018. DOI: http://dx.doi.org/10.1590/0034-7167.2014670503. 
13. Souza NVDO, Santos DM, Ramos EL, Anunciação CT, Thiengo PCS, Fernandes MC. Repercussões psicofísicas na saúde dos enfermeiros da adaptação e improvisação de materiais hospitalares. Esc. Anna Nery vol.14 no.2 Rio de Janeiro Apr./June 2010. Disponível em: <http://www.scielo.br/scielo.php?script=sci_arttext\&pid=S1414-81452010000200005>. Acesso em: 28 de setembro de 2018. DOI: http://dx.doi.org/10.1590/S141481452010000200005

14. Pacheco ES, Sousa ARR, Sousa PTM e Rocha AF. Prevalência dos sintomas osteomusculares relacionados ao trabalho de enfermagem no âmbito hospitalar. Rev Enferm UFPI. 2016 Oct-Dec;5(4):31-7. Disponível em: <http://www.ojs.ufpi.br/index.php/reufpi/article/view/5387>. Acesso em: 28 de setembro de 2018. DOI: https://doi. org/10.26694/reufpi.v5i4.5387

15. Nowak NL, Campos GA, Borba EO, Ulbricht L e Neves, EB. Fatores de risco para acidentes com materiais Perfurocortantes. O Mundo da Saúde, São Paulo - 2013;37(4):419-426. Disponível em: < https://www.saocamilo-sp. br/pdf/mundo_saude/155558/A06.pdf>. Acesso em: 28 de setembro de 2018.

16. Prata GP, Passos JP. A produção do conhecimento dos enfermeiros em saúde do trabalhador acerca do Cenário hospitalar. Rev. de Pesq.: cuidado é fundamental Online 2009. set/dez. 1(2): 255-264. Disponível em: <http:// www.redalyc.org/html/5057/505750816024/index.html>. Acesso em: 28 de setembro 2018. 